\title{
Laboratory Test Date Time of Reference Timepoint
}

National Cancer Institute

\section{Source}

National Cancer Institute. Laboratory Test Date Time of Reference Timepoint. NCI

Thesaurus. Code C87894.

The date and time of a laboratory test reference point. 